Viscoelastic Phase Separation Model for Ternary Polymer Solutions

\title{
Viscoelastic Phase Separation Model for Ternary Polymer Solutions
}

\author{
Kenji Yoshimoto ${ }^{1}$ and Takashi Taniguchi ${ }^{1, \text { a) }}$ \\ Department of Chemical Engineering, Kyoto University, Kyoto 615-8510, Japan
}

(Dated: 22 February 2021)

\begin{abstract}
When a polymer solution undergoes viscoelastic phase separation, the polymer-rich phase forms a networklike structure even if it is a minor phase. This unique feature is induced by polymer dynamics, which are constrained by the temporal entanglement of polymer chains. The fundamental mechanisms of viscoelastic phase separation have already been elucidated by theory and experiments over the past few decades; however, it is not yet well understood how viscoelastic phase separation occurs in multicomponent polymer solutions. Here, we construct a new viscoelastic phase separation model for ternary polymer solutions that consist of a polymer, solvent, and nonsolvent. Our simulation results reveal that a network-like structure is formed in the ternary bulk system through a phase separation mechanism similar to that observed in binary polymer solutions. A difference in dynamics is also found in that the solvent, whose affinity to the polymer is similar to that to the nonsolvent, moves freely between the polymer-rich and water-rich phases during phase separation. These findings are considered important for understanding the phase separation mechanism of ternary mixtures often used in the manufacturing of polymeric separation membranes.
\end{abstract}

PACS numbers: 82.60. Lf, 83.80. Rs, 83.60. Bc

Keywords: Polymer, Ternary Solution, Phase Separation, Viscoelastic, Model, Simulations

\section{INTRODUCTION}

When a binary liquid mixture undergoes phase separation, the minor component usually forms droplets in the matrix of the major component. However, if the mixture is composed of solvent and polymer and if the polymer chains are long enough to be entangled, the polymer solution may exhibit unique phase separation, termed viscoelastic phase separation. ${ }^{1-3}$ For example, in the early stage of viscoelastic phase separation, the major component, i.e. , the solvent, forms small droplets. ${ }^{4,5}$ In the late stage, the polymer-rich phase forms a networklike continuous structure even if it is the minor phase. ${ }^{6}$ These characteristics are due to a large difference in dynamic behavior between the solvent and the polymer. ${ }^{4}$ In the case of a binary liquid, each component diffuses down the concentration gradient, with speeds nearly the same between the two components. In viscoelastic phase separation, however, the entangled polymer chains move much more slowly than the solvent. In addition, the dynamic mechanical response differs between the polymer and the solvent. The entangled polymer deforms instantaneously as an elastic body does but also flows like a liquid over a period much longer than the relaxation time. These differences, which are referred to as dynamic asymmetries, may affect the mechanism and morphology of phase separation through tight coupling with concentration diffusion. ${ }^{2,4}$

In addition to the binary polymer solution, phase separation of the ternary polymer solution, which consists of polymer, solvent, and nonsolvent (e.g., water), is particularly important when preparing a polymer membrane. For example, in the nonsolvent-induced phase separation

\footnotetext{
a) Electronic mail: taniguchi@cheme.kyoto-u.ac.jp
}

(NIPS) process, which is widely used for the manufacture of polymer membranes, the polymer is dissolved in a solvent that is soluble both in the polymer and in water. By immersing the polymer solution in water, the solvent is partially replaced by the water. The resulting ternary polymer solution is thermodynamically unstable due to a strong repulsion between the water and the polymer and immediately separates into water-rich (= major) and polymer-rich (= minor) phases. The water-rich phase forms small droplets that become pores, while the polymer-rich phase forms a network-like structure as a matrix. Both phases are essentially similar to those observed in the viscoelastic phase separation of a binary polymer solution.

To provide insight into the phase separation mechanism in the NIPS process, some phase separation models have been proposed for the ternary polymer solution. ${ }^{7-9}$ Note, however, that the viscoelastic nature of the entangled polymer is not explicitly considered in any models. For example, the free energy of the ternary polymer solution has been described as a sum of the Flory-Huggins mixing energy and the interfacial energy, neglecting the elastic energy generated by deformation of the entangled polymer. Additionally, the relaxation of the entangled polymer is not considered in the existing models. Indeed, it has been demonstrated with binary polymer solutions that both elastic and relaxation behavior of the entangled polymer plays a crucial role in the formation of the frozen polymer-rich phase at the early stage and of the networklike polymer-rich structure at the late stage. $3,6,10,11$

In this paper, we develop a basic model for the phase separation of a ternary polymer solution that explicitly includes the elastic and relaxation characteristics of the entangled polymer. Our approach is based on the theory of viscoelastic phase separation developed for binary polymer solutions. ${ }^{1,6,10,11}$ First, we describe the free en- 
ergy of the ternary polymer solution as a functional of the volume fractions of each component and the deformation tensor of the entangled polymer chains (Sec. I A and I B). Then, we prepare a set of equations for evolving the volume fractions and the deformation tensor over time (Sec. I C). The coupling between the volume fractions and the deformation tensor occurs through the polymer velocity, which is determined from a balance among the thermodynamic, hydrodynamic, and elastic forces. Three different expressions for the polymer velocity are examined: a thermodynamic term only (diffusion case), thermodynamic + hydrodynamic terms (viscous case), and thermodynamic + hydrodynamic + elastic terms (viscoelastic case) (Sec. II). The first two cases are representatives of the previous models, and the last case is the focus of development in this study. Numerical calculations are performed to simulate the spinodal decomposition of a bulk ternary polymer solution and the change in morphology of a square-shaped polymer-rich phase immersed into a water-rich bath. By comparing the results obtained from the three cases, we highlight some important aspects of the viscoelasticity of an entangled polymer on the phase separation of a ternary polymer solution.

\section{A. Field variables: $\phi$ and $W$}

A ternary mixture of polymer, solvent, and water is described by three volume fractions: $\phi_{\mathrm{p}}, \phi_{\mathrm{s}}$, and $\phi_{\mathrm{w}}$. The subscripts p, s, and w denote polymer, solvent, and water, respectively. Each volume fraction varies with position $\boldsymbol{r}$ and time $t$, whereas the sum of the three volume fractions is always unity due to incompressibility;

$$
\sum_{\alpha=\mathrm{p}, \mathrm{s}, \mathrm{w}} \phi_{\alpha}=1
$$

In this paper, subscript $\alpha$ (or $\beta$ ) is used to denote one of the three components: polymer, solvent, and water.

Polymer chains are assumed to be long enough to have temporal entanglements, as schematically illustrated in Fig. 1. The entangled polymer chains form a temporal network structure that behaves like an elastic object for instantaneous, infinitesimal deformation. In contrast, the network structure flows like liquid under a constant load as a result of the stretch and orientation relaxations of entangled polymer chains by reptation motion and a constraint release mechanism. ${ }^{1}$ Such viscoelastic behavior of polymer entanglements can be included in the model by introducing another field variable, the so-called conformation tensor $\boldsymbol{W} .{ }^{6}$ A microscopic definition of $\boldsymbol{W}$ is given as follows. Let us consider an infinitesimal volume element that contains $n_{\mathrm{p}}(\gg 1)$ polymer chains. As schematically shown on the right-hand side of Fig. 1, all polymer chains are assumed to be Gaussian composed of $N$ statistical segments with a size of $a$. The $\ell$-th polymer chain $\left(\ell=1, \cdots, n_{\mathrm{p}}\right)$ has $Z_{\ell}(\gg 1)$ entangled segments whose positions are denoted by $\boldsymbol{r}_{1}^{\ell}, \ldots, \boldsymbol{r}_{Z_{\ell}}^{\ell}$. The vectors connecting two successive entangled segments,

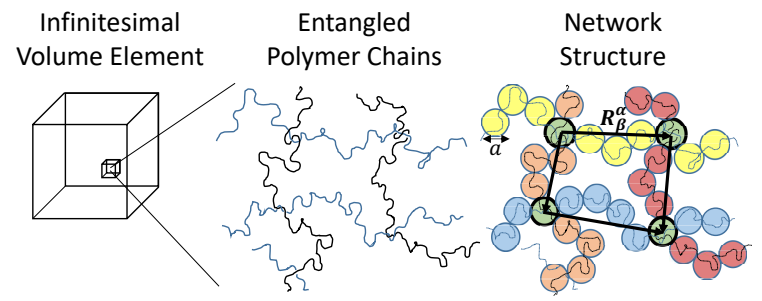

FIG. 1. Schematic representation of the temporal network structure of entangled polymer chains.

$\boldsymbol{R}_{m}^{\ell}\left(=\boldsymbol{r}_{m+1}^{\ell}-\boldsymbol{r}_{m}^{\ell}\right.$, where $\left.m=1, \ldots, Z_{\ell}-1\right)$, are considered as a frame of the network structure. At equilibrium, the average length of $\boldsymbol{R}_{m}^{\ell}$ is $a \sqrt{N_{\mathrm{e}}}$, where $N_{\mathrm{e}}$ is the average number of segments existing between the two adjacent entangled segments along a chain. The normalized dyadic tensor $\boldsymbol{R}_{m}^{\ell} \boldsymbol{R}_{m}^{\ell} / a^{2} N_{\mathrm{e}}$ represents the change in shape of the network structure from equilibrium. The average of $\boldsymbol{R}_{m}^{\ell} \boldsymbol{R}_{m}^{\ell} / a^{2} N_{\mathrm{e}}$ over all the entangled segments and chains existing in the same infinitesimal volume element is defined as the conformation tensor $\boldsymbol{W}$;

$$
\boldsymbol{W} \equiv \frac{1}{n_{\mathrm{p}}}\left[\sum_{\ell=1}^{n_{\mathrm{p}}} \frac{1}{\left(Z_{\ell}-1\right)} \sum_{m=1}^{Z_{\ell}-1} \frac{\boldsymbol{R}_{m}^{\ell} \boldsymbol{R}_{m}^{\ell}}{3 a^{2} N_{\mathrm{e}}}\right] .
$$

The right-hand side of Eq. (2) becomes an identity tensor $\boldsymbol{I}$ at equilibrium. Eq. (2) provides microscopic insight into the origin of polymer viscoelasticity. However, calculation of $\boldsymbol{W}$ from Eq. (2) is computationally demanding since it requires an explicit description of all polymer chains and entanglements. Alternatively, one can treat $\boldsymbol{W}$ as a continuous field variable and evolve it through a constitutive equation. ${ }^{12}$ In this approach, microscopic details of the polymer chains are discarded, whereas any local deformation of the network structure can be described by the same macroscopic constitutive relation arising from numerous entanglements of polymer chains. As described below, we take the latter approach, treating $\boldsymbol{W}$ as a continuous, frame-invariant deformation tensor and evolving $\boldsymbol{W}$ by a simple viscoelastic constitutive equation. ${ }^{12}$

\section{B. Thermodynamic properties}

Hereafter, we make all variables and equations dimensionless to generalize the model. For a ternary system with constant temperature $T$ and volume $V$, the free energy $F$ can be described as a functional of the volume fractions and conformation tensor;

$$
F\left[\phi_{\mathrm{p}}, \phi_{\mathrm{s}}, \phi_{\mathrm{w}}, \boldsymbol{W}\right]=\int_{V} d \boldsymbol{r}\left(f_{\mathrm{mix}}+f_{\text {int }}+f_{\text {ela }}\right),
$$

where $\phi_{\mathrm{w}}=1-\phi_{\mathrm{p}}-\phi_{\mathrm{s}}$. The first integrand in the right-hand side of Eq. (3), $f_{\text {mix }}$, represents a mixing free 
energy as ${ }^{13}$

$$
\begin{aligned}
f_{\text {mix }}= & \frac{\phi_{\mathrm{p}}}{N} \ln \phi_{\mathrm{p}}+\phi_{\mathrm{s}} \ln \phi_{\mathrm{s}}+\phi_{\mathrm{w}} \ln \phi_{\mathrm{w}} \\
& +\chi_{\mathrm{ps}} \phi_{\mathrm{p}} \phi_{\mathrm{s}}+\chi_{\mathrm{sw}} \phi_{\mathrm{s}} \phi_{\mathrm{w}}+\chi_{\mathrm{wp}} \phi_{\mathrm{w}} \phi_{\mathrm{p}},
\end{aligned}
$$

where $\chi_{\alpha \beta}$ is the Flory-Huggins parameter for the affinity between two different components, $\alpha$ and $\beta(\neq \alpha)$. The second integrand $f_{\text {int }}$ is the interfacial energy given by

$$
\begin{aligned}
f_{\text {int }}= & \frac{\kappa_{\mathrm{ps}}}{2}\left|\nabla\left(\phi_{\mathrm{p}}-\phi_{\mathrm{s}}\right)\right|^{2}+\frac{\kappa_{\mathrm{sw}}}{2}\left|\nabla\left(\phi_{\mathrm{s}}-\phi_{\mathrm{w}}\right)\right|^{2} \\
& +\frac{\kappa_{\mathrm{wp}}}{2}\left|\nabla\left(\phi_{\mathrm{w}}-\phi_{\mathrm{p}}\right)\right|^{2},
\end{aligned}
$$

where $\kappa_{\alpha \beta}$ is an interfacial parameter for a pair of components $\alpha$ and $\beta(\neq \alpha)$. The last integrand $f_{\text {ela }}$ represents elastic energy arising from deformation of the temporal network composed of entangled polymer chains. Among several different formulae proposed for $f_{\text {ela }}$, we select the one that contains only the shear modulus of the polymer solution; ${ }^{6}$

$$
f_{\text {ela }}=\frac{G}{4}(\boldsymbol{W}-\boldsymbol{I}):(\boldsymbol{W}-\boldsymbol{I}),
$$

where $\boldsymbol{I}$ is the identity tensor. The shear modulus $G$ is assumed to vary with the polymer concentration based on the scaling theory; ${ }^{14}$

$$
G=G_{0} \phi_{\mathrm{p}}^{3}
$$

The scaling of Eq. (7) stems from the characteristic length between the adjacent entangled segments, $\xi$. According to the book of de Gennes ${ }^{15}$, the shear modulus of entangled polymer solution can be expressed as $G \sim k_{\mathrm{B}} T / \xi^{3}$. Since $\xi$ scales with $1 / \phi_{\mathrm{p}}, G$ is inversely proportional to $\phi_{\mathrm{p}}^{3}$ as shown in Eq. (7). The coefficient $G_{0}$ will be used as a parameter to characterize the elasticity of the polymer solution.

The chemical potential $\mu_{\alpha}(\alpha=\mathrm{p}, \mathrm{s})$ and the stress tensor $\sigma$ are derived from the functional derivative of the free energy $F$ in Eq. (3); ${ }^{16}$

$$
\begin{aligned}
\mu_{\alpha} & =\frac{\delta F}{\delta \phi_{\alpha}}=\frac{\partial f}{\partial \phi_{\alpha}}-\nabla \cdot \frac{\partial f}{\partial\left(\nabla \phi_{\alpha}\right)}, \\
\boldsymbol{\sigma} & =2 \boldsymbol{W} \cdot \frac{\delta F}{\delta \boldsymbol{W}}=2 G \boldsymbol{W} \cdot(\boldsymbol{W}-\boldsymbol{I}),
\end{aligned}
$$

where $f \equiv f_{\text {mix }}+f_{\text {int }}+f_{\text {ela }}$. The detailed expressions of $\mu_{\alpha}$ are summarized in Appendix A.

\section{Time evolution of the field variables}

The volume fractions are evolved with the equations of continuity;

$$
\frac{\partial \phi_{\mathrm{p}}}{\partial t}=-\boldsymbol{\nabla} \cdot\left(\phi_{\mathrm{p}} \boldsymbol{v}_{\mathrm{p}}\right), \quad \frac{\partial \phi_{\mathrm{s}}}{\partial t}=-\boldsymbol{\nabla} \cdot\left(\phi_{\mathrm{s}} \boldsymbol{v}_{\mathrm{s}}\right) .
$$

Note that $\phi_{\mathrm{w}}$ is determined by the compressibility condition, Eq. (1). In this study, the random noise term arising from the thermal fluctuations ${ }^{17}$ is not included in Eq. (10) so as to clarify the viscoelastic effects on the domain dynamics at the late stage of phase separation. In general, the thermal fluctuations have little influence on the domain dynamics after the formation of sharp interfaces ${ }^{18}$. The velocities of the polymer and solvent, $\boldsymbol{v}_{\mathrm{p}}$ and $\boldsymbol{v}_{\mathrm{s}}$, respectively, can be obtained by solving the equations of motion derived based on the concept of stress division $^{19}$. The resulting expressions are written as

$$
\begin{aligned}
& \boldsymbol{v}_{\mathrm{p}}=\boldsymbol{v}-\frac{1}{\phi_{\mathrm{p}}}\left\{L_{\mathrm{pp}}\left(\nabla \mu_{\mathrm{p}}-\frac{\boldsymbol{\nabla} \cdot \boldsymbol{\sigma}}{\phi_{\mathrm{p}}}\right)+L_{\mathrm{ps}} \boldsymbol{\nabla} \mu_{\mathrm{s}}\right\}, \\
& \boldsymbol{v}_{\mathrm{s}}=\boldsymbol{v}-\frac{1}{\phi_{\mathrm{s}}}\left\{L_{\mathrm{sp}}\left(\nabla \mu_{\mathrm{s}}-\frac{\boldsymbol{\nabla} \cdot \boldsymbol{\sigma}}{\phi_{\mathrm{s}}}\right)+L_{\mathrm{ss}} \boldsymbol{\nabla} \mu_{\mathrm{s}}\right\}
\end{aligned}
$$

where $L_{\alpha \beta}$ is the component of the transport coefficient matrix $\boldsymbol{L}$ and where $\boldsymbol{v}$ is the mean velocity defined as

$$
\boldsymbol{v}=\boldsymbol{v}_{\mathrm{p}} \phi_{\mathrm{p}}+\boldsymbol{v}_{\mathrm{s}} \phi_{\mathrm{s}}+\boldsymbol{v}_{\mathrm{w}} \phi_{\mathrm{w}}
$$

The mean velocity $\boldsymbol{v}$ satisfies the Stokes equation;

$$
0=-\nabla p-\sum_{i=\mathrm{p}, \mathrm{s}} \phi_{i} \nabla \mu_{i}+\eta \Delta \boldsymbol{v}+\boldsymbol{\nabla} \cdot \boldsymbol{\sigma},
$$

where $p$ and $\eta$ denote the pressure and viscosity of the ternary mixture, respectively. In this study, we simplify Eqs. (11) and (12) by assuming that the diagonal components of $\boldsymbol{L}$ are constants and the off-diagonal components are zero;

$$
\begin{aligned}
& \boldsymbol{v}_{\mathrm{p}}=\boldsymbol{v}-\frac{L_{\mathrm{pp}}}{\phi_{\mathrm{p}}}\left(\nabla \mu_{\mathrm{p}}-\frac{\nabla \cdot \boldsymbol{\sigma}}{\phi_{\mathrm{p}}}\right), \\
& \boldsymbol{v}_{\mathrm{s}}=\boldsymbol{v}-\frac{L_{\mathrm{ss}}}{\phi_{\mathrm{s}}} \nabla \mu_{\mathrm{s}} .
\end{aligned}
$$

Equation (15) has exactly the same expression as that derived for a polymer-solvent binary system. ${ }^{6}$ A new addition to the ternary system is Eq. (16), which allows the solvent to move separately from the polymer.

To evolve the conformation tensor simultaneously with the volume fractions described above, we employ a constitutive equation, the so-called upper-convected Maxwell equation, ${ }^{12}$

$$
\frac{D \boldsymbol{W}}{D t}=\left(\boldsymbol{\nabla} \boldsymbol{v}_{\mathrm{p}}\right) \cdot \boldsymbol{W}+\boldsymbol{W} \cdot\left(\boldsymbol{\nabla} \boldsymbol{v}_{\mathrm{p}}\right)^{\mathrm{T}}-\frac{1}{\tau}(\boldsymbol{W}-\boldsymbol{I}),
$$

where $D / D t$ represents the substantial derivative $\left(=\partial / \partial t+\boldsymbol{v}_{\mathrm{p}} \cdot \boldsymbol{\nabla}\right)$ and $\left(\boldsymbol{\nabla} \boldsymbol{v}_{\mathrm{p}}\right)^{\mathrm{T}}$ denotes the transpose of the velocity gradient tensor. The relaxation time $\tau$ is assumed to have the same dependency on $\phi_{\mathrm{p}}$ as the shear modulus defined in Eq. (7);

$$
\tau=\tau_{0} \phi_{\mathrm{p}}^{3}
$$

with a constant $\tau_{0}$. 
TABLE I. Simulation parameters.

\begin{tabular}{lc}
\hline \hline Parameter & Value \\
\hline Number of statistical segments per chain: $N$ & 10 \\
Flory-Huggins parameters: $\chi_{\mathrm{ps}}, \chi_{\mathrm{sw}}, \chi_{\mathrm{wp}}$ & $0.4,0.3,2.3$ \\
Initial volume fractions: $\phi_{\mathrm{p} 0}, \phi_{\mathrm{s} 0}, \phi_{\mathrm{w} 0}$ & $0.15,0.60,0.25$ \\
Interfacial parameters: $\kappa_{\mathrm{ps}}, \kappa_{\mathrm{sw}}, \kappa_{\mathrm{wp}}$ & $1.0,1.0,1.0$ \\
Grid size: $d$ & 2.0 \\
Box length: $D$ & 512 \\
Time step: $\Delta t$ & 0.025 \\
Transport coefficients: $L_{\mathrm{pp}}, L_{\mathrm{ss}}$ & $1.0,1.0$ \\
Viscoelastic parameters: $\tau_{0}, G_{0}, \eta$ & $10,100,0.1$ \\
\hline \hline
\end{tabular}

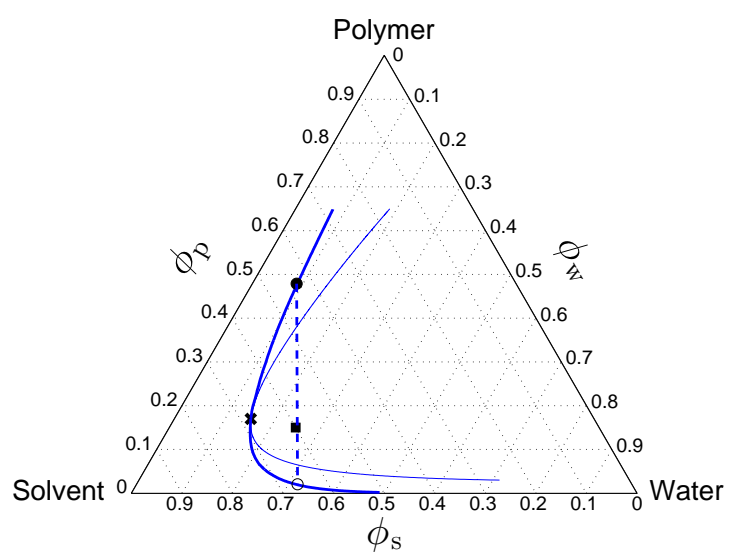

FIG. 2. Phase diagram for the ternary system. The thin and thick lines represent the spinodal and binodal curves, respectively, and they merge on the critical point $(x)$. The simulation starts from a homogeneously mixed state $(\boldsymbol{\square})$ and eventually reaches equilibrium composed of polymer-rich $(\bullet)$ and water-rich (०) phases. The final compositions of these phases can be connected with a straight line (dashed line) passing through the initial composition.

(a)

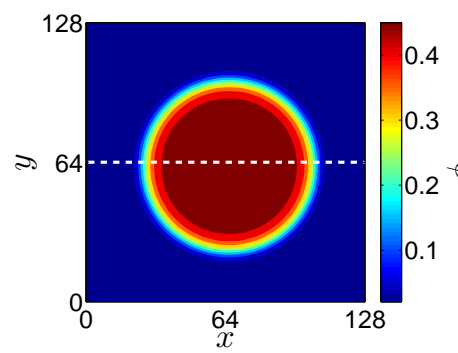

(b)

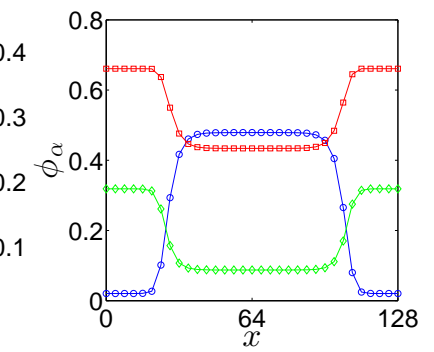

FIG. 3. (color online) Polymer-rich domain at equilibrium. (a) Two-dimensional plot of the polymer volume fraction $\phi_{\mathrm{p}}$. Color is assigned to each square grid based on the value of $\phi_{\mathrm{p}}$. For example, red and blue colors represent polymer-rich and water-rich phases, respectively. (b) Profile of the volume fraction along the dashed line in (a). All three volume fractions, $\phi_{\mathrm{p}}, \phi_{\mathrm{s}}$, and $\phi_{\mathrm{w}}$, are shown here with the symbols of circle, square, and diamond, respectively.

\section{SIMULATIONS}

\section{A. Parameters}

The parameters used in the simulations are summarized in Table I. The Flory-Huggins parameters $\chi_{\alpha \beta}$ are chosen from the literature ${ }^{20}$ to represent a ternary mixture composed of polyvinylidene difluoride (PVDF), dimethylformamide (DMF), and water. Note that $\chi_{\mathrm{ps}}$ and $\chi_{\mathrm{sw}}$ depend on the concentration. ${ }^{20}$ However, for brevity, they are assumed to be constant. The number of statistical segments per chain, $N$, is set at a relatively small number, 10, to increase numerical stability and to perform large-scale simulations.

Figure 2 illustrates a phase diagram of the ternary system with $N$ and $\chi_{\alpha \beta}$ given in Table I. The binodal and spinodal curves are drawn using the mixing free energy density given in Eq. (4). The initial polymer volume fraction, $\phi_{\mathrm{p} 0}$ is set to be 0.15 , which a typical value used in the NIPS process ${ }^{20}$. The initial volume fraction of the water, $\phi_{\mathrm{w} 0}$, is arbitrary chosen to be 0.25 , assuming that $\sim 30 \%$ of the solvent in the bulk polymer solution is instantaneously substituted with the water. With the given initial composition, the ternary bulk system is already located at the inside of spinodal region so that it is spontaneously decomposed into polymer-rich and waterrich phases. The final compositions of the two phases, $\phi_{\alpha, \text { eq }}^{\prime}$ and $\phi_{\alpha, \text { eq }}^{\prime \prime}$, are obtained from the fully equilibrated morphology shown in Fig. 3.

The interfacial parameters $\kappa_{\alpha \beta}$ are set arbitrarily to 1.0. Then, the grid size $d$ is adjusted to 2.0 , with which the interfacial regions are expressed by 5 or 6 grid points [see Fig. 3(b)]. For the time-related parameters, the transport coefficients, $L_{\mathrm{pp}}$ and $L_{\mathrm{ss}}$, are set to unity. Then, the time step $\Delta t$ is increased to the maximum, 0.025 , with which the iterative calculations can be performed stably over a long course of simulation time.

Finally, the two viscoelastic parameters, $\tau_{0}$ and $G_{0}$, are set to 10 and 100, respectively. The viscosity of the ternary system, $\eta$, is set at 0.1 , which is estimated from $\eta=G \tau$ with $\phi_{\mathrm{p}}=\phi_{\mathrm{p} 0}$.

\section{B. Three typical cases}

To grasp some fundamental behaviors of phase separation in the ternary system, we perform simulations for the following three cases. The same equations and parameters are used in all cases, except that expressions for the polymer and solvent velocities are modified depending on the cases.

(I) Diffusion case. The velocity of component $\alpha(=\mathrm{p}, \mathrm{s})$ is induced only by the gradient of the chemical potential of the same component;

$$
\boldsymbol{v}_{\alpha}=-\left(L_{\alpha} / \phi_{\alpha}\right) \nabla \mu_{\alpha} .
$$

Neither the mean velocity $\boldsymbol{v}$ nor the stress tensor $\boldsymbol{\sigma}$ is calculated here. 
(II) Viscous case. In addition to case (I), the hydrodynamic contribution is taken into account;

$$
\boldsymbol{v}_{\alpha}=\boldsymbol{v}-\left(L_{\alpha \alpha} / \phi_{\alpha}\right) \nabla \mu_{\alpha} .
$$

The Stokes equation, Eq. (14) is used to solve $\boldsymbol{v}$, but the elastic contribution, $\boldsymbol{\nabla} \cdot \boldsymbol{\sigma}$, is excluded.

(III) Viscoelastic case. The polymer velocity is expressed by Eq. (15), including all the diffusion, hydrodynamic, and elastic contributions. The solvent velocity is represented by Eq. (16) which is identical to Eq. (20).

\section{RESULTS AND DISCUSSION}

\section{A. Phase separation in ternary mixtures}

Figure 4 illustrates the distributions of the polymer volume fraction $\phi_{\mathrm{p}}$ obtained at different times. All simulations are started from a homogeneous mixture, as shown on the leftmost image. Uniform random noise ranging between -0.025 and 0.025 is added to the initial volume fraction, whereas it is too small to be visible on the leftmost image.

In case (I), a bicontinuous structure appears vaguely at $t=100$ and becomes clearer at $t=250$. This stage is referred to as the linear (or early) stage of spinodal decomposition, where the polymer-rich and waterrich phases become denser while maintaining their spatial frequencies. Once the polymer-rich phase is broken into pieces through further densification and local shrinkage $(t=500)$, smaller pieces of the polymer-rich phase are gradually absorbed into larger domains by an evaporation-condensation mechanism $(t>500)$.

In case (II), the morphology change observed in the early stage of spinodal decomposition is almost identical to that in case (I). A noticeable difference can be found at $t=500$, where the polymer-rich domains become larger and more rounded than those in case (I). This is because the deformation of the polymer-rich domains is accelerated by hydrodynamic flow (see Sec. III B for the details of the hydrodynamic effects). Once the discretization and circularization of the polymer-rich domains is settled $(t=1000)$, the hydrodynamic flow is diminished, and therefore, the morphology change is significantly slowed down. Later, the polymer-rich domains are gradually coarsened through the evaporation-condensation mechanism, similar to case (I).

In case (III), a bicontinuous structure is clearly seen at $t=2500$, which is slower than the other two cases by a factor of 10. It is also noteworthy that the time scale of this delay is much longer than the relaxation time $\tau_{0}$, i.e., 10. In the viscoelastic phase separation, whenever the polymer-rich domain is deformed by the thermodynamic force, the elastic force is instantaneously generated as a counterforce. Then the residual and new thermodynamic force acts again on the polymer-rich domain, generating the new elastic force in the polymer-rich domain (see Sec. III-B for the detailed mechanisms). Since this cycle occurs iteratively and continuously throughout the entire phase separation process, it takes a considerably long time for the polymer-rich domain to be fully relaxed. Meanwhile, the solvent and water are gradually squeezed out from the polymer-rich phase. As a result, a network-like structure is formed with irregularly shaped polymer-rich domains $(t=5000)$. Some frameworks of the network-like structure are maintained over time, whereas others are merged into larger ones $(t>5000)$. The latter is driven by a hydrodynamic flow that is caused by disconnection of sharper edges and/or thinner parts of the network-like structure. In all three cases, the water-rich phase is formed simultaneously with the polymer-rich phase. As illustrated in Fig. 5 , the water distributes inversely to the polymer, reflecting the fact that the water is strongly repelled by the polymer $\left(\chi_{\mathrm{wp}}=2.3\right)$. The water flows in the direction of thickening the water-rich phase, similar to the polymer moving towards the thicker domains. On the other hand, the solvent distributes over the system and moves across the polymer-rich and water-rich phases. This is mainly because the affinity between the solvent and water $\left(\chi_{\mathrm{sw}}=0.3\right)$ is almost the same as that between the solvent and polymer $\left(\chi_{\mathrm{ps}}=0.4\right)$. A similar flow trend can be observed at $t=250$ in cases (I) and (II) (results are not shown here due to space restriction). Although the solvent's free movement does not change any basic features of the phase separation, it becomes particularly important for the NIPS process where the solvent needs to move across the interface between the polymer solution and the water ${ }^{7,9,20-22}$.

In the rest of the paper, we focus on the analysis of polymer-rich phases to clarify some important roles of polymer viscoelasticity in phase separation in ternary systems.

The morphology change can be quantified with the characteristic wavenumber $\bar{q}$ defined as $^{23}$

$$
\bar{q}=\frac{\int d \boldsymbol{q}|\boldsymbol{q}| S(\boldsymbol{q})}{\int d \boldsymbol{q} S(\boldsymbol{q})}
$$

where $\boldsymbol{q}$ is the wave vector. The structure factor $S(\boldsymbol{q})$ is obtained from the square of the Fourier transform of the polymer's volume fraction, i.e., $\left\langle\left|\hat{\phi}_{\mathrm{p}}(\boldsymbol{q})\right|^{2}\right\rangle$, where $\langle(\cdots)\rangle$ stands for the statistical average of $(\cdots)$. Figure 6 shows $\bar{q}$ of the polymer morphology sampled over a time period of $2.5 \times 10^{4}$ (including the images in Fig. 4). In case (I), $\bar{q}$ is almost constant at $t<300$, and then it decreases monotonically as $\bar{q} \propto t^{-1 / 3}$. The former corresponds to the initial stage of spinodal decomposition, and the latter indicates the domain growth driven by the evaporation-condensation mechanism. ${ }^{6}$ In case (II), a sharp decrease in $\bar{q}$ is observed from $t=300$ to $t=800$, with a rate of $\bar{q} \propto t^{-2 / 3}$. The exponent of $-2 / 3$ has also been observed in the binary polymer solution ${ }^{6}$ and fluid mixture ${ }^{24}$ where the numerical simulations were performed in two dimensions including the hydrodynamic effects. Note, however, that the hydrodynamic-driven domain growth generally scales with $t^{-1} \cdot{ }^{25,26}$ This dis- 


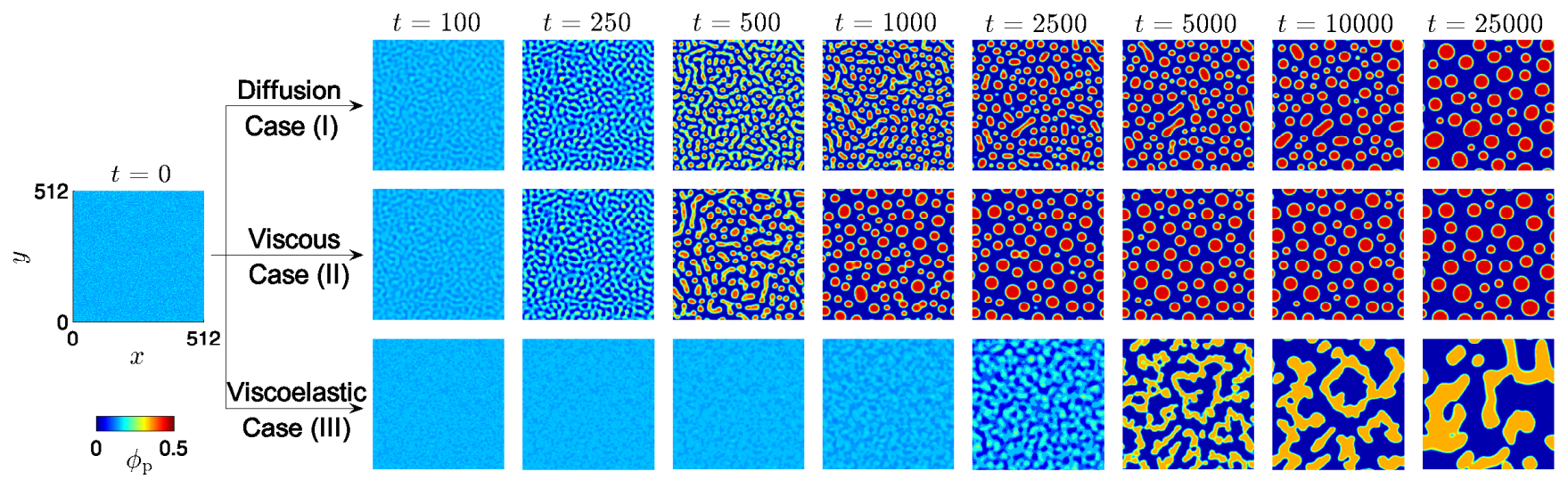

FIG. 4. (color online) Phase separation in the bulk ternary system. Each image represents a two-dimensional distribution of polymer volume fraction $\phi_{\mathrm{p}}$ within the simulation box of size $L_{\mathrm{box}} \times L_{\mathrm{box}}=512^{2}$. The color is assigned based on the value of $\phi_{\mathrm{p}}$, as shown in the scale bar.
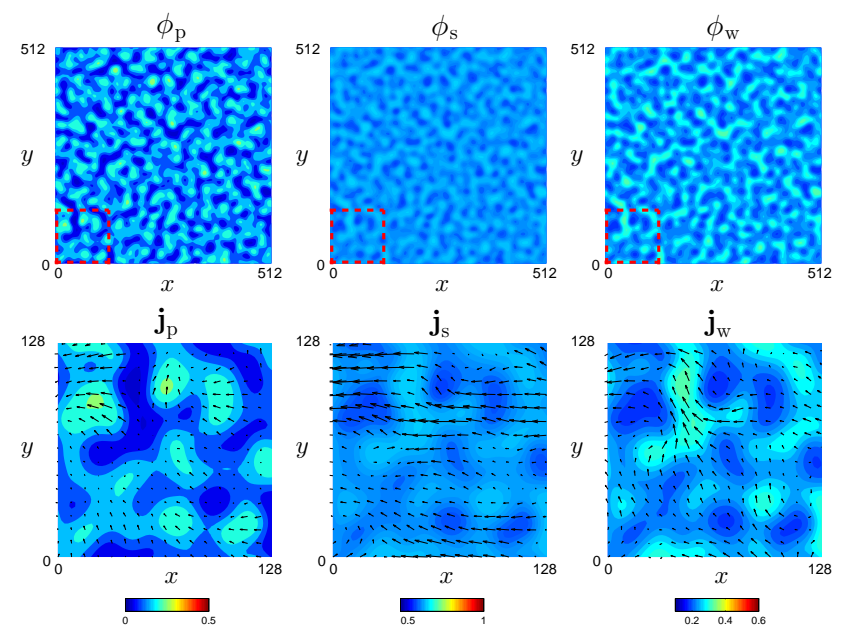

FIG. 5. (color online) Morphology and flow of each component at an early stage of phase separation in case (III) $(t=2500)$ : (top) two-dimensional distribution of the volume fraction $\phi_{\alpha}(\alpha=\mathrm{p}, \mathrm{s}, \mathrm{w})$, and (bottom) volumetric flux $\boldsymbol{j}_{\alpha}$ within the area enclosed by dashed lines in the top image. A color is assigned to each grid point based on the value of $\phi_{\alpha}$. Arrows on the bottom images represent a vector field of $\boldsymbol{j}_{\alpha}$, whose lengths are magnified by $3 \times 10^{3}$ for clarity.

crepancy is possibly due to the difference in dimension, the limitation of system size and time scale, or the combination of the hydrodynamic and diffusion effects ${ }^{25,27}$. In the later time, $\bar{q}$ remains almost unchanged with the disappearance of the hydrodynamic flow. At $t>10^{4}$, $\bar{q}$ follows the same trend as case (I), indicating that the polymer-rich domains regrow under the evaporationcondensation mechanism. In case (III), $\bar{q}$ shows a slight decrease over time, followed by a sharp drop as $\bar{q} \propto t^{-2 / 3}$ at $t>3000$. The exponent of $-2 / 3$ indicates the domain growth driven by the hydrodynamic flow. The hydrodynamic flow in case (III) is induced after breaking off some

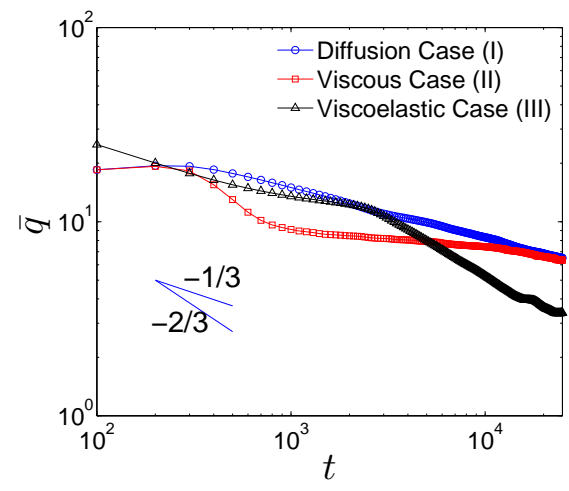

FIG. 6. (color online) Change in the characteristic wavenumber $\bar{q}$ over time $t$.

unstable parts in the network-like structure.

Figure 7 illustrates a time evolution of the circularly averaged structure factor $S(q)$ ( $q$ : wavenumber) at an early stage of spinodal decomposition, calculated from

$$
S(q)=\frac{1}{2 \pi q d q} \int_{|\boldsymbol{q}|=q}^{|\boldsymbol{q}|=q+d q} d \boldsymbol{q}\left\langle\left|\hat{\phi}_{\mathrm{p}}(\boldsymbol{q})\right|^{2}\right\rangle .
$$

In cases (I) and (II), $S(q)$ shows a peak at $q_{\max } \approx 20$. The peak height $S_{\max }$ increases exponentially over time, which is one of the typical characteristics in the early stage of spinodal decomposition. Note that $q_{\max }$ can be estimated numerically by solving the eigenequations derived in Appendix B. In case (III), no sharp peak is formed, indicating that the phase separation is suppressed by the elastic effects. Another feature of case (III) is that $S(q)$ decreases over time at $q>q_{\max }$. This is due to smoothing of the random noise added to the initial volume fractions. An opposite trend is seen in cases (I) and (II) since the random noise is already diminished and the interface is sharpened over time.

In addition to the morphological change, the phase compositions also change differently in the three cases. 

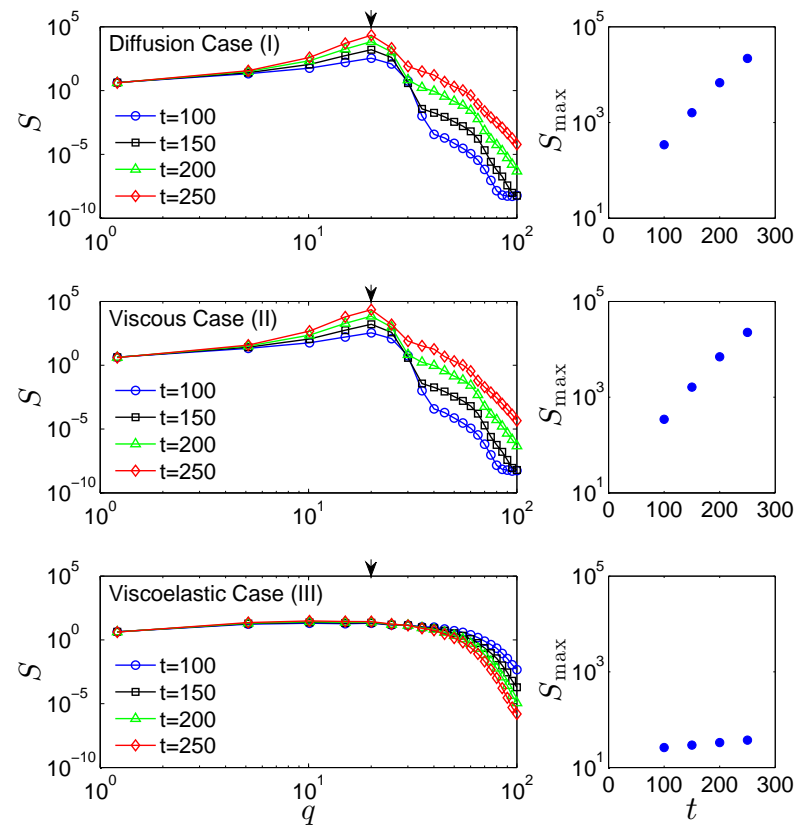

FIG. 7. (color online) Structure factor $S$ at the early stage of spinodal decomposition. (Left) A log-log plot of $S$ as a function of the wavenumber $q$. The arrow points $q=20$ (= $\left.q_{\max }\right)$, where $S$ reaches the maximum, $S_{\max }$, in cases (I) and (II). (Right) A semilog graph of $S_{\max }$ as a function of time $t$.

Figure 8 illustrates the polymer volume fraction averaged over the polymer-rich domains $\left(\phi_{\mathrm{p}}>\phi_{\mathrm{p} 0}\right)$ and the polymer volume fraction averaged over the water-rich domains $\left(\phi_{\mathrm{p}}<\phi_{\mathrm{p} 0}\right)$, denoted by $\phi_{\mathrm{p}}^{\prime}$ and $\phi_{\mathrm{p}}^{\prime \prime}$, respectively. The interfacial regions are excluded from the averaging. In the early stage of spinodal decomposition, $\phi_{\mathrm{p}}^{\prime}$ and $\phi_{\mathrm{p}}^{\prime \prime}$ of case (I) overlap with those of case (II). In the later stage, $\phi_{\mathrm{p}}^{\prime}$ and $\phi_{\mathrm{p}}^{\prime \prime}$ in case (I) asymptotically approach the equilibrium values, $\phi_{\mathrm{p}, \mathrm{eq}}^{\prime}$ and $\phi_{\mathrm{p}, \text { eq }}^{\prime \prime}$, respectively (see Fig. 3 for the values of $\phi_{\mathrm{p}, \mathrm{eq}}^{\prime}$ and $\left.\phi_{\mathrm{p}, \mathrm{eq}}^{\prime \prime}\right)$. On the other hand, in case (II), the phase compositions reach equilibrium almost ten times faster than in case (I). In case (III), $\phi_{\mathrm{p}}^{\prime}$ and $\phi_{\mathrm{p}}^{\prime \prime}$ are constrained to the initial value $\phi_{\mathrm{p} 0}(=0.15)$ over a relatively long period, and then they exhibit a sharp change similar to those in the early stage of cases (I) and (II). All these trends are consistent with those observed in Figs. 4-7, except that in case (III), $\phi_{\mathrm{p}}^{\prime}$ settles into a lower value $(0.35)$ than $\phi_{\mathrm{p}, \text { eq }}^{\prime}(0.48)$. This can also be seen as a color difference in the polymer-rich domains at $t>2500$ in Fig. 4. The less densified polymer-rich domains are induced by the elastic force, which always acts against the thermodynamic force (see next section for the details of the two forces). It should also be noted that in the plateau regime of case (III), $\phi_{\mathrm{p}}^{\prime}$ still keeps increasing at a considerably slow rate; it will take an extremely long time for $\phi_{\mathrm{p}}^{\prime}$ to reach to $\phi_{\mathrm{p}, \mathrm{eq}}^{\prime}$.

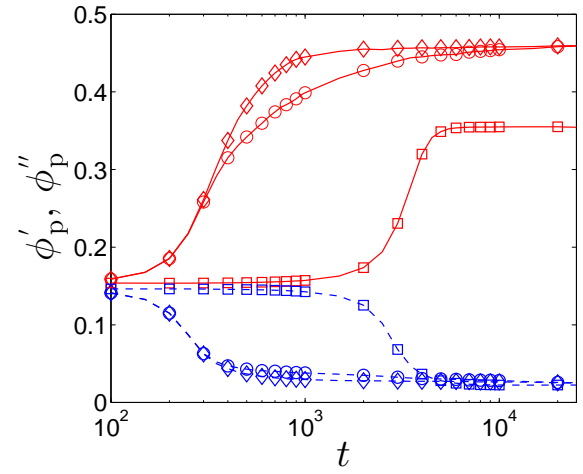

FIG. 8. (color online) Averaged polymer volume fractions in the polymer-rich phase $\left(\phi_{\mathrm{p}}^{\prime}\right)$ and in the water-rich phase $\left(\phi_{\mathrm{p}}^{\prime \prime}\right)$ : circle for diffusion case (I), diamond for viscous case (II), and square for viscoelastic case (III). The solid and dashed lines correspond to $\phi_{\mathrm{p}}^{\prime}$ and $\phi_{\mathrm{p}}^{\prime \prime}$, respectively.

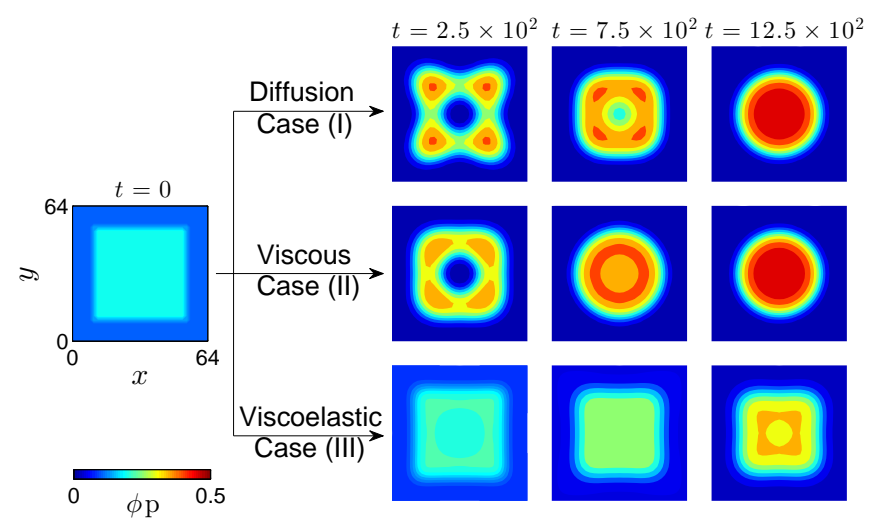

FIG. 9. (color online) Change in shape of the polymer-rich phase. For all cases, the polymer-rich phase is set to be a square at $t=0$, as shown on the leftmost image. Each image represents a two-dimensional distribution of $\phi_{\mathrm{p}}$ within the simulation box of $L_{\text {box }} \times L_{\text {box }}=64^{2}$. Colors are assigned based on the scale bar.

\section{B. Driving forces for morphology change}

To clarify a relationship between the morphology change of the polymer-rich domains and the polymer velocity $\boldsymbol{v}_{\mathrm{p}}$, we set up another initial condition, as illustrated in the leftmost image in Fig. 9. The compositions of the polymer-rich and water-rich phases, $\phi_{\alpha}^{\prime}$ and $\phi_{\alpha}^{\prime \prime}$ ( $\alpha=\mathrm{p}, \mathrm{s}, \mathrm{w})$, are estimated from the bicontinuous structure formed at $t=2500$ in case (III): $\left(\phi_{\mathrm{p}}^{\prime}, \phi_{\mathrm{s}}^{\prime}, \phi_{\mathrm{w}}^{\prime}\right)=$ $(0.194,0.584,0.222)$, and $\left(\phi_{\mathrm{p}}^{\prime \prime}, \phi_{\mathrm{s}}^{\prime \prime}, \phi_{\mathrm{w}}^{\prime \prime}\right)=(0.102,0.617$, 0.281 ). The size of the square-shaped polymer-rich phase is determined in a way that the averaged volume fractions over the system become equal to the initial volume fractions used in the previous section, $\phi_{\alpha 0}$. With this setting, $\phi_{\mathrm{p}}^{\prime}$ and $\phi_{\mathrm{p}}^{\prime \prime}$ eventually reach $\phi_{\mathrm{p}, \text { eq }}^{\prime}$ and $\phi_{\mathrm{p}, \text { eq }}^{\prime \prime}$, as seen in Fig. 8.

Figure 9 shows the polymer morphology at $t=0,250$, 


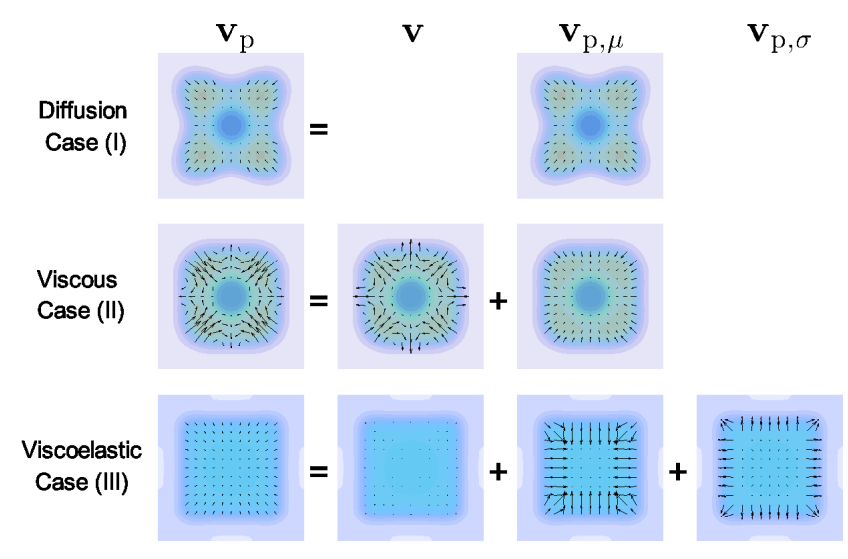

FIG. 10. (color online) Polymer velocity $\mathbf{v}_{\mathrm{p}}$ in the polymerrich phase at $t=2500$ in Fig. 9. The velocity is represented by arrows on top of the distribution of $\phi_{\mathrm{p}}$. The velocity field on the leftmost image corresponds to $\mathbf{v}_{\mathrm{p}}$, and the ones on the second, third, and fourth left columns represent the mean velocity $\mathbf{v}$ (not calculated in case (I)), the polymer velocity driven by the gradient of chemical potential, $\mathbf{v}_{\mathrm{p}, \mu}$, and that driven by the divergence of the stress tensor, $\mathbf{v}_{\mathrm{p}, \sigma}$ (calculated only in case (III)), respectively. For clarity, the number of arrows is reduced from $32 \times 32$ to $16 \times 16$, and only those located inside of the polymer-rich phase $\left(\phi_{\mathrm{p}}>0.15\right)$ are shown here. All arrows are magnified uniformly by 200; however, some of them are still too small to be apparent.

750, and 1250 for all three cases. In case (I), the polymer initially moves towards the corners of the square since the solvent and water diffuse out more from the corners than from the middle edges. As the corners of the square become rich in polymer, the interfaces become concave, and the inside becomes hollow $(t=250)$. The polymer movement is plotted in Fig. 10, where the majority of the polymer velocity $\mathbf{v}_{\mathrm{p}}$ points to the polymer-rich corner regions. Note that in case (I), $\mathbf{v}_{\mathrm{p}}$ is equal to the velocity driven by the gradient of the polymer potential, $\mathbf{v}_{\mathrm{p}, \mu}\left(=-L_{\mathrm{pp}} / \phi_{\mathrm{p}} \boldsymbol{\nabla} \mu_{\mathrm{p}}\right)$, as defined in Eq. (19). The polymer-rich phase constantly exudes the solvent and water until it reaches equilibrium. As a result, the polymerrich phase is continuously shrunk and transformed into a circular shape $(t=1250)$ through a rounded square shape $(t=750)$. In case (II), the morphology change occurs similarly to that in case (I); however, the corners of the polymer-rich square become rounded earlier than in case (I). This is mainly due to the hydrodynamic flow, which is not considered in case (I). As defined in Eq. (20), the polymer velocity $\mathbf{v}_{\mathrm{p}}$ in case (II) is composed of the mean velocity $\mathbf{v}$ and the diffusion-driven velocity $\mathbf{v}_{\mathrm{p}, \mu}$. For instance, each velocity at $t=250$ can be seen in Fig. 10. Similar to case (I), $\mathbf{v}_{\mathrm{p}, \mu}$ points to the polymer-rich corners. On the other hand, v exhibits a large flow that enhances the rounding of the polymerrich phase. Once the polymer-rich phase is transformed into a circular shape $(t=750)$, the hydrodynamic effect is diminished, and the change in the composition of
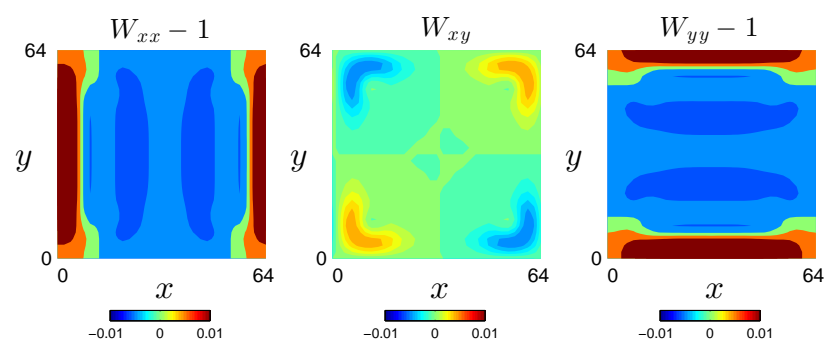

FIG. 11. (color online) Conformation tensor $\boldsymbol{W}$ at $t=2500$ : (left) $W_{x x}-1$, (center) $W_{x y}$, and (right) $W_{y y}-1$. A color is assigned using the scale bar on the bottom side. The negative (or positive) regions in $W_{i i}-1(i=x$ or $y)$ indicate where the polymer is locally compressed (or stretched) along the $i$ direction.

the polymer-rich phase is governed by the same diffusion process as in case (I). In case (III), the square shape of the polymer-rich phase is maintained even at $t=12500$. As expressed in Eq. (15), $\mathbf{v}_{\mathrm{p}}$ in case (III) is the sum of $\mathbf{v}, \mathbf{v}_{\mathrm{p}, \mu}$, and the velocity driven by the divergence of the stress tensor, $\mathbf{v}_{\mathrm{p}, \sigma}\left(=L_{\mathrm{pp}} / \phi_{\mathrm{p}}^{2} \boldsymbol{\nabla} \cdot \boldsymbol{\sigma}\right)$. Notably, the flow of $\mathbf{v}_{\mathrm{p}, \sigma}$ in Fig. 10 is almost perfectly opposite to that of $\mathbf{v}_{\mathrm{p}, \mu}$. This can be interpreted as a balance between the externally acting thermodynamic force and the internally generated elastic force. Indeed, the polymer-rich phase is under internal compression, as shown in Fig. 11; the elastic force is generated to counteract the compressive strain. Note that according to the Stokes equation as shown in Eq. (14), $\mathbf{v}$ is induced only by solvent movement when $\mathbf{v}_{\mathrm{p}, \mu}$ is completely offset by $\mathbf{v}_{\mathrm{p}, \sigma}$. Therefore, $\mathbf{v}$ in case (III) becomes much smaller than in case (II), resulting in a large reduction in any polymer movements.

The magnitude of the polymer velocity, $\left|\mathbf{v}_{\mathrm{p}}\right|$, as shown in Fig. 12, is calculated by taking an average of the magnitudes of the polymer velocity within the polymer-rich phase $\left(\phi_{\mathrm{p}}>0.15\right)$. In case $(\mathrm{I}),\left|\mathbf{v}_{\mathrm{p}}\right|$ in the early stage shows a gradual decrease over time $(100 \leq t \leq 250)$, subsequently remaining almost unchanged $(250 \leq t \leq 750)$. The former corresponds to a slowdown of the polymer movement towards the corners, and the latter corresponds to a shrinkage of the polymer-rich phase driven by diffusion out of the solvent and water. The composition of the polymer-rich phase reaches equilibrium during a sharp drop of $\left|\mathbf{v}_{\mathrm{p}}\right|(t \geq 750)$. In case (II), two plateaus are observed: one from $t=100$ to $t=200$ and another from $t=400$ to $t=600$. The first plateau is associated with the rounding of the polymer-rich phase driven by hydrodynamic flow. The second plateau overlaps with $\left|\mathbf{v}_{\mathrm{p}}\right|$ in case (I), indicating that the morphology change of the polymer-rich phase is driven by the diffusion process, similar to case (I). In case (III), the initial value of $\left|\mathbf{v}_{\mathrm{p}}\right|$ is approximately 5 times smaller than that of cases (I) and (II) due to the elastic effects mentioned above. Since the elastic force is constantly generated to balance out the thermodynamic force, $\left|\mathbf{v}_{\mathrm{p}}\right|$ remains the same or- 


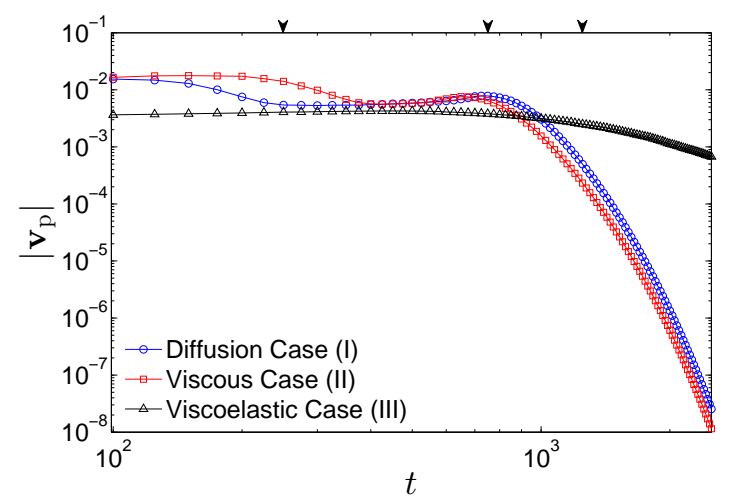

FIG. 12. (color online) Change in the magnitude of the polymer velocity, $\left|\mathbf{v}_{\mathrm{p}}\right|$, over time $t$. The arrows on the top $x$-axis indicate where the data of Fig. 10 are sampled.

der of magnitude over the entire simulation time. This explains why the morphology of the polymer-rich phase remains square, keeping the polymer volume fraction relatively low inside.

\section{CONCLUSION}

We constructed a new model for the viscoelastic phase separation of ternary polymer solutions consisting of entangled polymers, solvents, and nonsolvents. The effects of both elasticity and relaxation of the entangled polymer were incorporated into the model by adding elastic energy to the free energy of the bulk ternary system and by introducing relaxation time into the constitutive equation. Our numerical simulations of the bulk ternary polymer solution demonstrated that the polymer-rich phase was frozen at the early stage of viscoelastic phase separation, whereas it was transformed into a network-like structure and gradually relaxed over a long time at the late stage. Both phenomena were essentially the same as those observed in the binary polymer solution. A new finding was that the solvent, which was soluble to the polymer and the nonsolvent, moved across the polymerrich and water-rich phases during the viscoelastic phase separation of the polymer ternary solution. As a final note, we simplified our model by setting some model parameters (e.g., mobility coefficients $L_{\alpha \beta}$ and the viscosity $\eta$ ) to be constant to clarify the viscoelastic effects of the entangled polymer on ternary phase separation. In reality, these parameters vary with the concentration and affect the phase separation to some degree. Indeed, some previous studies showed in simulations that the concentration dependency of $L_{\alpha \beta}$ and $\eta$ could be crucial for the formation of the glassy polymerrich domains ${ }^{28}$ and asymmetric polymeric membranes formed in the NIPS process ${ }^{9,21,22}$. It should be emphasized here that both viscous and elastic features of the polymer solution are fundamentally essential for the viscoelastic phase separation; a simple addition of the concentration-dependent parameters into the diffusion or viscous model may not be sufficient to reproduce the frozen and recovering features of the viscoelastic phase separation simultaneously. We are currently investigating the combinatory effect of the viscoelasticity of the entangled polymer, the concentration-dependent model parameters, and the thermal fluctuations on the phase separation of ternary polymer solutions.

\section{ACKNOWLEDGMENTS}

This work was partially supported by JSPS KAKENHI Grant Number 19H01862. We also acknowledge the supercomputer systems of the Institute for Chemical Research and Academic Center for Computing and Media Studies, Kyoto University.

\section{Data Availability}

The data that supports the findings of this study are available within the article.

${ }^{1}$ M. Doi and S. F. Edwards, The Theory of Polymer Dynamics (Oxford University Press, 1986).

${ }^{2}$ H. Tanaka, J. Phys. Condens. Matter 12, 207 (2000).

${ }^{3}$ T. Taniguchi, J. Soc. Rheol. Jpn. 32, 27 (2004).

${ }^{4}$ H. Tanaka, Phys. Rev. Lett. 76, 787 (1996).

${ }^{5}$ A. Onuki and T. Taniguchi, J. Chem. Phys. 106, 5761 (1997).

${ }^{6}$ T. Taniguchi and A. Onuki, Phys. Rev. Lett. 77, 4910 (1996).

${ }^{7}$ B. Zhou and A. C. Powell, J. Membr. Sci. 268, 150 (2006).

${ }^{8}$ Y. Mino, T. Ishigami, Y. Kagawa, and H. Matsuyama, J. Membr. Sci 483, 104 (2015).

${ }^{9}$ D. R. Tree, K. T. Delaney, H. D. Ceniceros, T. Iwama, and G. H. Fredrickson, Soft Matter 13, 3013 (2017).

${ }^{10}$ H. Tanaka, Phys. Rev. E 56, 4451 (1997).

${ }^{11}$ T. Araki and H. Tanaka, Macromolecules 34, 1953 (2001).

${ }^{12}$ R. G. Larson, Constitutive Equations for Polymer Melts and Solutions (Butterworth-Heinemann, 2013).

${ }^{13}$ P. J. Flory, J. Chem. Phys 10, 51 (1942).

${ }^{14}$ A. Onuki, J. Phys. Soc. Jpn. 59, 3423 (1990).

${ }^{15}$ P.-G. de Gennes, Scaling Concepts in Polymer Physics (Cornell Univ. Press, 1979)

${ }^{16}$ S. T. Milner, Phys. Rev. E 48, 3674 (1993).

${ }^{17}$ K. Fukawatase, K. Yoshimoto, and M. Ohshima, Jpn. J. Appl. Phys. 54, 06FE01 (2015).

${ }^{18}$ G. Brown and A. Chakrabarti, J. Chem. Phys. 98, 2451 (1993).

${ }^{19}$ A. Onuki, J. Non-Cryst. Solids 172, 1151 (1994).

${ }^{20}$ H. Matsuyama, M. Teramoto, R. Nakatani, and T. Maki, J. Appl. Polym. Sci. 74, 159 (1999).

${ }^{21}$ D. R. Tree, T. Iwama, K. T. Delaney, J. Lee, and G. H. Fredrickson, ACS Macro Lett. 7, 582 (2018).

22 J. U. Garcia, T. Iwama, E. Y. Chan, D. R. Tree, K. T. Delaney, and G. H. Fredrickson, ACS Macro Lett. 9, 1617 (2020).

${ }^{23}$ H. Tanaka and T. Araki, Chem. Eng. Sci. 61, 2108 (2006).

${ }^{24}$ H. Furukawa, Phys. Rev. E 55, 1150 (1997).

${ }^{25}$ E. D. Siggia, Phys. Rev. A 20, 595 (1979).

${ }^{26}$ T. Koga, K. Kawasaki, M. Takenaka, and T. Hashimoto, Physica A 198, 473 (1993)

${ }^{27}$ E. Scholten, L. M. C. Sagis, and E. van der Linden, Macromolecules 38, 3515 (2005).

${ }^{28}$ B. F. Barton, P. D. Graham, and A. J. McHugh, Macromolecules 31, 1672 (1998).

${ }^{29}$ Y. Tanaka, I. Noda, and M. Nagawasa, Macromolecules 18, 2220 (1985).

${ }^{30}$ Y. Tanaka, M. Umeda, and I. Noda, Macromolecules 21, 2257 (1988). 


\section{Appendix A: Chemical potential}

The chemical potentials $\mu_{\mathrm{p}}$ and $\mu_{\mathrm{s}}$ are expressed as

$$
\mu_{\mathrm{p}}=\mu_{\mathrm{p}}^{*}-\mu_{\mathrm{w}}^{*}, \quad \mu_{\mathrm{s}}=\mu_{\mathrm{s}}^{*}-\mu_{\mathrm{w}}^{*},
$$

where

$$
\begin{aligned}
\mu_{\mathrm{p}}^{*}= & \frac{1}{N}\left(\ln \phi_{\mathrm{p}}+1\right)+\chi_{\mathrm{ps}} \phi_{\mathrm{s}}+\chi_{\mathrm{wp}} \phi_{\mathrm{w}} \\
& -\kappa_{\mathrm{ps}}\left(\Delta \phi_{\mathrm{p}}-\Delta \phi_{\mathrm{s}}\right)-\kappa_{\mathrm{wp}}\left(\Delta \phi_{\mathrm{p}}-\Delta \phi_{\mathrm{w}}\right) \\
& +\frac{3}{4} G_{0} \phi_{\mathrm{p}}^{2}(\boldsymbol{W}-\boldsymbol{I}):(\boldsymbol{W}-\boldsymbol{I}), \\
\mu_{\mathrm{s}}^{*}= & \ln \phi_{\mathrm{s}}+1+\chi_{\mathrm{ps}} \phi_{\mathrm{p}}+\chi_{\mathrm{sw}} \phi_{\mathrm{w}} \\
& -\kappa_{\mathrm{ps}}\left(\Delta \phi_{\mathrm{s}}-\Delta \phi_{\mathrm{p}}\right)-\kappa_{\mathrm{sw}}\left(\Delta \phi_{\mathrm{s}}-\Delta \phi_{\mathrm{w}}\right), \\
\mu_{\mathrm{w}}^{*}= & \ln \phi_{\mathrm{w}}+1+\chi_{\mathrm{sw}} \phi_{\mathrm{s}}+\chi_{\mathrm{wp}} \phi_{\mathrm{p}} \\
& -\kappa_{\mathrm{sw}}\left(\Delta \phi_{\mathrm{w}}-\Delta \phi_{\mathrm{s}}\right)-\kappa_{\mathrm{wp}}\left(\Delta \phi_{\mathrm{w}}-\Delta \phi_{\mathrm{p}}\right) .
\end{aligned}
$$

\section{Appendix B: Analysis of the characteristic wavelength in ternary spinodal decomposition}

Here, we assume that ternary phase separation occurs by a simple diffusion mechanism, as in case (I). The continuity equation for the polymer is written as

$$
\begin{aligned}
\frac{\partial \phi_{\mathrm{p}}}{\partial t} & =L_{\mathrm{pp}} \boldsymbol{\nabla} \cdot\left[\boldsymbol{\nabla} \frac{\delta F}{\delta \phi_{\mathrm{p}}}\right] \\
& =L_{\mathrm{pp}} \Delta\left(h_{\mathrm{p}}+K_{\mathrm{p} 1} \Delta \phi_{\mathrm{p}}+K_{\mathrm{p} 2} \Delta \phi_{\mathrm{s}}\right)
\end{aligned}
$$

where

$$
\begin{aligned}
h_{\mathrm{p}} & \left.\approx \frac{\partial f_{\mathrm{mix}}}{\partial \phi_{\mathrm{p}}}\right|_{\phi_{\alpha 0}}+\left.\frac{\partial^{2} f_{\mathrm{mix}}}{\partial \phi_{\mathrm{p}}^{2}}\right|_{\phi_{\alpha 0}}\left(\phi_{\mathrm{p}}-\phi_{\mathrm{p} 0}\right), \\
K_{\mathrm{pp}} & =-\kappa_{\mathrm{ps}}-\kappa_{\mathrm{sw}}+4 \kappa_{\mathrm{wp}}, \\
K_{\mathrm{ps}} & =\kappa_{\mathrm{ps}}^{2}-\kappa_{\mathrm{sw}}+2 \kappa_{\mathrm{wp}} .
\end{aligned}
$$

At the early stage, each volume fraction deviates slightly from the initial values;

$$
\phi_{\alpha}(\boldsymbol{r}, t)=\phi_{\alpha 0}+u_{\alpha}(\boldsymbol{r}, t)
$$

where $u_{\alpha}(\boldsymbol{r}, t)(\alpha=\mathrm{p}, \mathrm{s})$ denotes a small perturbation. By substituting Eq. (B5) into Eq. (B1), the polymer continuity equation is expressed with respect to $u_{\mathrm{p}}$;

$$
\frac{\partial u_{\mathrm{p}}}{\partial t}=L_{\mathrm{pp}} \Delta\left(\left.\frac{\partial^{2} f_{\mathrm{mix}}}{\partial \phi_{\mathrm{p}}^{2}}\right|_{\phi_{\alpha 0}} u_{\mathrm{p}}+\sum_{\alpha=\mathrm{p}, \mathrm{s}} K_{\mathrm{p} \alpha} \Delta u_{\alpha}\right)
$$

Similarly, the continuity equation for the solvent can be described as

$$
\frac{\partial u_{\mathrm{s}}}{\partial t}=L_{\mathrm{ss}} \Delta\left(\left.\frac{\partial^{2} f_{\mathrm{mix}}}{\partial \phi_{\mathrm{s}}^{2}}\right|_{\phi_{\alpha 0}} u_{\mathrm{s}}+\sum_{\alpha=\mathrm{p}, \mathrm{s}} K_{\mathrm{s} \alpha} \Delta u_{\alpha}\right)
$$

where

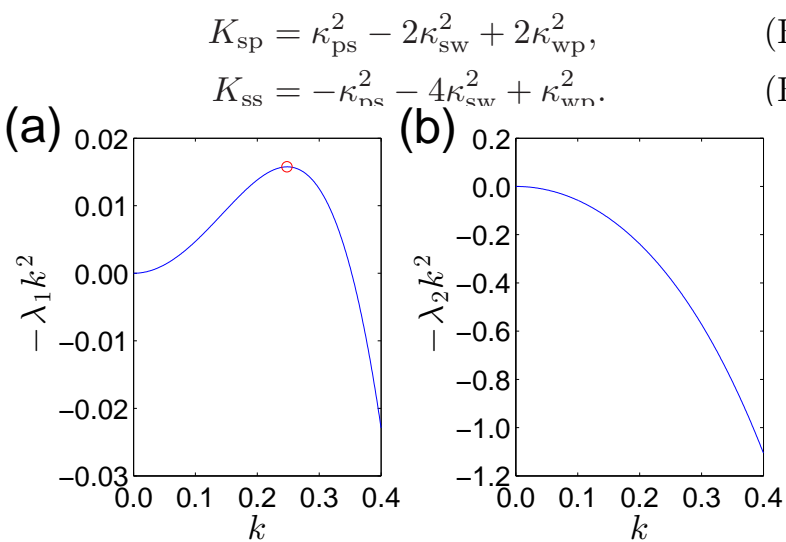

FIG. 13. Frequency-dependent coefficient $-\lambda k^{2}$ : (a) $-\lambda_{1} k^{2}$ and (b) $-\lambda_{2} k^{2}$. The circular mark on (a) denotes the maximum point, $(0.248,0.0158)$.

After taking the Fourier transform, Eqs. (B6) and (B7) can be written in matrix form as

$$
\frac{d}{d t}\left[\begin{array}{c}
\hat{u}_{\mathrm{p}} \\
\hat{u}_{\mathrm{s}}
\end{array}\right]=-k^{2} \boldsymbol{\Omega}\left[\begin{array}{c}
\hat{u}_{\mathrm{p}} \\
\hat{u}_{\mathrm{s}}
\end{array}\right]
$$

where $k$ is the angular frequency $\left(=2 \pi q / L_{\text {box }}\right)$ and $\hat{u}_{\alpha}$ is the Fourier transform of $u_{\alpha}$. The matrix $\Omega$ is defined as

$$
\boldsymbol{\Omega}=\left[\begin{array}{cc}
L_{\mathrm{pp}}\left(\left.\frac{\partial^{2} f_{\mathrm{mix}}}{\partial \phi_{\mathrm{p}}^{2}}\right|_{\phi_{\alpha 0}}-K_{\mathrm{pp}} k^{2}\right) & -L_{\mathrm{pp}} K_{\mathrm{ps}} k^{2} \\
L_{\mathrm{ss}}\left(\left.\frac{\partial^{2} f_{\mathrm{mix}}}{\partial \phi_{\mathrm{s}}^{2}}\right|_{\phi_{\alpha 0}}-K_{\mathrm{sp}} k^{2}\right) & -L_{\mathrm{ss}} K_{\mathrm{ss}} k^{2}
\end{array}\right] .
$$

The eigenvalues of $\Omega, \lambda$, can be obtained by solving

$$
\operatorname{det}|\boldsymbol{\Omega}-\lambda \boldsymbol{I}|=0 \text {, }
$$

which can be negative or positive depending on the value of $k$. In the eigenspace, $\boldsymbol{\Omega}$ on the right-hand side of Eq. (B10) can be replaced with a diagonal matrix composed of $\lambda$. Therefore, the sign of $-\lambda k^{2}$ is essential to determine whether fluctuation of the volume fractions at a given frequency $k$ is amplified or diminished over time. The two eigenvalues calculated from Eq. (B12) are plotted in Fig. 13. Here, $-\lambda_{1} k^{2}$ forms a positive peak at $k=0.248$ $\left(\equiv k_{\max }\right)$, whereas $-\lambda_{2} k^{2}$ always becomes negative at $k>0$. This indicates that the fluctuation of the volume fraction at $k=k_{\max }$ may be amplified at the fastest rate. Note that the wavenumber corresponding to $k_{\max }$ is 20.2 ; the result here agrees with $q_{\max }$ as shown in Fig. 7. 